“C 2015 IEEE. Personal use of this material is permitted. Permission from IEEE must be obtained for all other uses, in any current or future media, including reprinting/republishing this material for advertising or promotional purposes, creating new collective works, for resale or redistribution to servers or lists, or reuse of any copyrighted component of this 


\title{
Recognizing emotions from EEG subbands using wavelet analysis
}

\author{
Henry Candra*, Mitchell Yuwono, Ardi Handojoseno, Rifai Chai, Steven Su, and Hung T. Nguyen
}

\begin{abstract}
Objectively recognizing emotions is a particularly important task to ensure that patients with emotional symptoms are given the appropriate treatments. The aim of this study was to develop an emotion recognition system using Electroencephalogram (EEG) signals to identify four emotions including happy, sad, angry, and relaxed. We approached this objective by firstly investigating the relevant EEG frequency band followed by deciding the appropriate feature extraction method. Two features were considered namely: 1. Wavelet Energy, and 2. Wavelet Entropy. EEG Channels reduction was then implemented to reduce the complexity of the features. The ground truth emotional states of each subject were inferred using Russel's circumplex model of emotion, that is, by mapping the subjectively reported degrees of valence (pleasure) and arousal to the appropriate emotions - for example, an emotion with high valence and high arousal is equivalent to a 'happy' emotional state, while low valence and low arousal is equivalent to a 'sad' emotional state. The Support Vector Machine (SVM) classifier was then used for mapping each feature vector into corresponding discrete emotions. The results presented in this study indicated that Wavelet features extracted from alpha, beta and gamma bands seem to provide the necessary information for describing the aforementioned emotions. Using the DEAP (Dataset for Emotion Analysis using electroencephalogram, Physiological and Video Signals), our proposed method achieved an average sensitivity and specificity of $77.4 \% \pm 14.1 \%$ and $69.1 \% \pm 12.8 \%$, respectively.
\end{abstract}

\section{INTRODUCTION}

Emotions as a part of non-verbal communication play an important role in interactions between individuals. Emotions are directly related to the brain and are manifested in the form of brain waves that affect the entire system of a person's body. This brain wave is known as electroencephalography (EEG) which is a type of physiological signal that has been implemented in various emotion recognition system schemes.

Psychologist James Russell proposed the circumplex model of emotion in 1980 [1]. The circumplex model is a conceptualized 2-dimensional continuous space where the horizontal and vertical axes correspond to the degree of valence (pleasure) and arousal, respectively. Discrete emotional states such as 'happy', 'sad', 'angry', and 'relaxed' can be inferred from the degree of valence and arousal as illustrated in Fig. 1. Using this model, the degree of any of the aforementioned discrete emotional state can be measured. It was further reported that the psychological condition of

*Henry Candra, Mitchell Yuwono, Ardi Handojoseno, Rifai Chai, Steven W. Su, and Hung. T. Nguyen are with the Centre for Health Technologies, Faculty of Engineering and Information Technology, University of Technology, Sydney, New South Wales, Australia (email: Henry.Candraestudent.uts.edu.au, mitchellyuwono@gmail.com, AluysiusMariaArdi. Handojoseno@student.uts.edu.au, (Rifai.Chai, Steven.Su, Hung.Nguyen) Quts.edu.au) positive/negative arousal (activation/deactivation) and positive/negative valence (pleasant/unpleasant) can be identified from Galvanic Skin Response (GSR) and EEG signal [2].

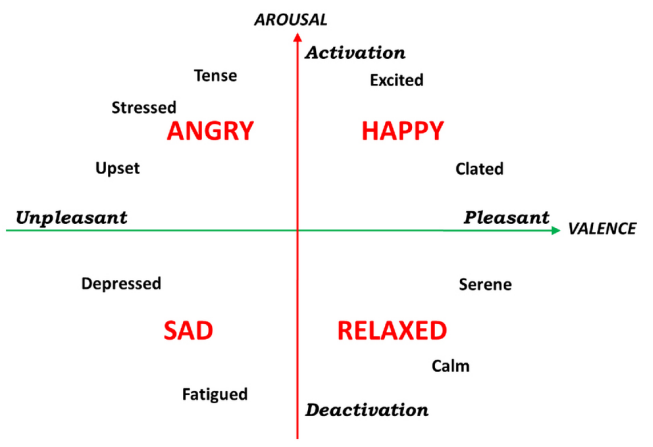

Fig. 1. Russell's circumplex model of affect [1]. Horizontal axis represents valence (pleasure); vertical axis represents arousal. Artwork is as seen in Valenza et. al [3].

Studies have indicated that the reliability of most EEGbased system can be improved by proper:

1) Feature selection To date, there is still no consensus as to which features are most suitable for EEG emotion recognition [4]. Two of the popular methods often considered are Fast Fourier Transform (FFT) [5] and Discrete Wavelet Transform (DWT) [6].

2) EEG subband selection: EEG waveforms are generally subdivided into several bandwidths known as delta, theta, alpha, beta, and gamma. These bands need to be properly selected as not all of them carry the relevant information for emotion recognition. For example, Zhu et. al. discovered that EEG patterns in the Beta and Gamma bands are generally stable across emotions and subjects [7].

3) EEG channel selection: Jenke suggested that not all of the electrode locations carries the appropriate information for emotions [4].

In this paper, we propose an EEG emotion recognition method to identify four discrete emotions namely: happy, sad, angry, and relaxed using wavelet features including energy and entropy. The classification algorithm used in this paper is the Multi-class Support Vector Machine (SVM) [8]. We also investigated the effect of EEG channel and subband selection.

On the DEAP (Dataset for Emotion Analysis using electroencephalogram, Physiological and Video Signals) [9], our method achieved the average sensitivity and specificity of $72.3 \%$ and $83.8 \%$ using the Wavelet Entropy features. We observed an increase of specificity from $79.8 \%$ to $82.1 \%$ 
using the alpha, beta, and gamma subbands. Further specificity increase to $83.8 \%$ was achieved using 18 EEG channels namely: Fp1, Fp2, AF3, AF4, F3,F4, F7, F8, FC5, FC6, T7, T8, P7, P8, P3, P4, O1, and O2. We did not observe any substantial decrease in sensitivity which gives a positive indication of the appropriateness of the selected channels and subbands.

\section{Materials, Method, AND Experimental OVERVIEW}

\section{A. Materials}

Our experiment was conducted using the DEAP [9]. DEAP recorded 32-channels EEG signals from 32 participants. This dataset was created by asking each participant to watch 40 music videos (one minute duration each) with their EEG recorded. As they watch, the participants subjectively rated their degree of valence and arousal from the scale of 1 to 9.

We then map this subjective rating into four discrete emotion class that can be inferred from the circumplex model as shown in Fig. 1 as follows:

- Pleasant + Activation $\Rightarrow$ Happy

- Pleasant + Deactivation $\Rightarrow$ Relaxed

- Unpleasant + Activation $\Rightarrow$ Angry

- Unpleasant + Deactivation $\Rightarrow \mathrm{Sad}$

The training and test sets are each composed of those signals from a group of 5 participants.

\section{B. Method Overview}

The block diagram of the method is shown in Fig. 2. The detailed overview is as follows.

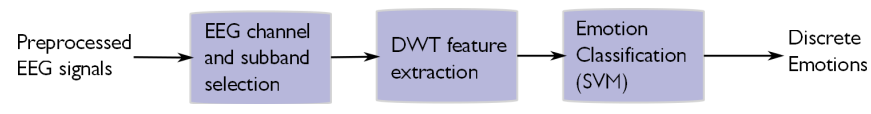

Fig. 2. Block diagram of the proposed system.

In order to map a raw EEG signal to the appropriate emotion, we employ the following steps:

- Preprocess the raw EEG signal to remove artifacts such as eye blinking and down-sampling the signal to $128 \mathrm{~Hz}$. The preprocessed DEAP dataset is readily available in the DEAP website [9].

- Compute the wavelet features using DWT from a given EEG channel and take the wavelet energy and wavelet entropy from the decomposition level that corresponds to the appropriate EEG subband.

\section{- Train the SVM classifier:}

- Generate the training and test sets:

* Perform signal preprocessing and DWT feature extraction for each emotion for each subject from a group of 5 participants and assign this array of observations as the training set, so that

$$
\mathbb{X}_{\text {train }}=\left\{\mathbb{X}_{1}, \mathbb{X}_{2}, \ldots, \mathbb{X}_{5}\right\}
$$

where $\mathbb{X}_{n}=\left\{\mathbf{x}_{1, n}, \mathbf{x}_{2, n}, \ldots\right\}$ denote the observation vectors extracted from participant $n$.
* Similarly, the test set $\mathbb{X}_{\text {test }}$ can then be constructed from another 5 participants so that

$$
\mathbb{X}_{\text {test }} \cap \mathbb{X}_{\text {train }}=\emptyset \text {. }
$$

- Optimize the support vector centroids and radius for the RBF kernel from $\mathbb{X}_{\text {train }}$ using the Ensemble Rapid Centroid Estimation (ERCE) [10].

- Train the multi-class SVM using $\mathbb{X}_{\text {train }}$ against the class labels. We used Sequential Minimal Optimization (SMO) algorithm for the SVM training algorithm.

\section{Experimental Overview}

1) EEG subband selection: We investigated two experimental settings which involved training the SVM using wavelet features extracted from the:

$\mathrm{SB}_{5}$ : 5 EEG frequency bands: delta, theta, alpha, beta, and gamma: $\mathrm{SB}_{5}=\{\delta, \theta, \alpha, \beta, \gamma\}$, and

$\mathrm{SB}_{3}$ : 3 EEG frequency bands: alpha, beta, and gamma: $\mathrm{SB}_{3}=\{\alpha, \beta, \gamma\}$.

According to Tinguely et. al, [11] the delta and theta band are more relevant to the sleep stage, thus has less relevance to the emergence of emotion.

2) EEG channel selection: We also experimented on the effect of channels selection as follows:

$\mathrm{CH}_{32}$ : No channel selection, i.e. using all the 32 available EEG channels.

$\mathrm{CH}_{18}$ : The number of channels was reduced to the 18 channels commonly used in the EEG emotion recognition literature according to Jenke et. al [4]. The 18 channels are: Fp1, Fp2, AF3, AF4, F3,F4, F7, F8, FC5, FC6, T7, T8, P7, P8, P3, P4, O1, and O2.

\section{METHOD}

\section{A. Discrete Wavelet Transform (DWT) Feature Extraction}

The DWT is advantageous in a sense that it provides timefrequency localization, multiscale zooming, and multirate filtering for detecting and characterizing transients. These advantages allow DWT to potentially extract the appropriate information from non-stationary signals such as the EEG signals [12].

Given any one dimensional time signal $x(t)$, the DWT decomposes $x(t)$ based on dyadic scales and positions defined as follows:

$$
\operatorname{DWT}(x(t) ; a, n)=\int_{-\infty}^{\infty} x(t) \frac{1}{\sqrt{2^{a}}} \psi\left(\frac{t-2^{a} n}{2^{a}}\right) d t
$$

where $2^{a} n$ and $2^{a}$ are the time localization and scale respectively, while $\psi(t)$ denotes the mother wavelet function.

The DWT can be interpreted as a filtering process using a dyadically shifted and scaled mother wavelet. Given a sampled signal $x[n]=x\left(n T_{s}\right)$ where $T_{s}$ denotes the time between samples, the DWT can then be computed recursively for each level of decomposition by convolving $x[n]$ with a quadrature mirror filter with high-pass impulse response $g[n]$ and low-pass impulse response $h[n]$ and downsampling the resulting signals by a factor of 2 . The resulting signals 
$x_{A}=(x * h)[n]$ and $x_{D}[n]=(x * g)[n]$ are referred to as the approximation and detail coefficients, respectively. Programmatically, the DWT can be defined as a recursive operation as follows:

$$
\begin{aligned}
& x_{A_{a+1}}[n] \leftarrow\left(x_{A_{a}} * g\right) \downarrow_{2} \stackrel{\text { def }}{=} \sum_{k=-\infty}^{\infty} x[k] g[2 n-k], \text { and } \\
& x_{D_{a+1}}[n] \leftarrow\left(x_{A_{a}} * h\right) \downarrow_{2} \stackrel{\text { def }}{=} \sum_{k=-\infty}^{\infty} x[k] h[2 n-k] .
\end{aligned}
$$

where $a$ denotes the level of decomposition, while $\downarrow_{2}$ denotes the downsampling operator by a factor of 2 .

1) Wavelet Energy: The wavelet energy $E(a)$ measures the energy of the wavelet coefficient localized at the $a^{\text {th }}$ level of decomposition computed as follows,

$$
E(a)=\left\|C_{a}\right\|^{2}=\sum_{n} C_{a}^{2}[n]
$$

where $C_{a}$ denotes the wavelet coefficients at the $a^{t h}$ decomposition level. $C_{a}$ can be either $x_{A_{a}}$ or $x_{D_{a}}$.

Normalizing the wavelet energy against the total wavelet energy, a probability mass function is obtained as follows,

$$
p(a)=\frac{E(a)}{\sum_{k=1}^{K} E(k)},
$$

where $K$ denotes the number of discrete wavelet decompositions, $p(a) \in\{0,1\}$ and $\sum_{a} p(a)=1$. This normalized measure is also known as the relative wavelet energy [13].

2) Wavelet Entropy: The wavelet entropy $H(a)$ measures the degree of 'unpredictability' in the energy distribution. Given the probability mass function $p(a)$ (refer to Eq. (5)), the wavelet entropy is calculated as follows [14],

$$
H(a)=-p(a) \log p(a),
$$

where $K$ denotes the number of DWT decompositions.

\section{B. Optimizing the Multi-class SVM Classifier}

The emotion classification is done using a multi-class SVM with a Radial Basis Function (RBF) kernel as follows,

$$
\mathrm{RBF}_{\mathrm{JS}}\left(\mathbf{x}, \boldsymbol{\mu}_{k}, \sigma_{k}\right)=\exp \left(-\frac{\mathrm{JS}\left(\mathbf{x} \| \boldsymbol{\mu}_{k}\right)}{2 \sigma_{k}^{2}}\right)
$$

where $k$ denotes the support vector index, $\sigma_{k}$ denotes the support vector radius, JS $\left(\mathbf{x} \| \boldsymbol{\mu}_{k}\right)$ denotes the Jensen-Shannon (JS) divergence between a random vector $\mathbf{x}$ and the support vector centroid $\boldsymbol{\mu}_{k}$.

The JS divergence is a symmetrized and smoothed version of the Kullback-Leibler (KL) divergence [15]. Given two discrete probability distributions $P \sim p(x)$ and $Q \sim q(x)$, the JS divergence is calculated as follows,

$$
\begin{aligned}
\mathrm{JS}(P \| Q) & =\frac{1}{2} \mathrm{KL}(P \| M)+\frac{1}{2} \mathrm{KL}(Q \| M), \text { where } \\
\mathrm{KL}(R \| S) & =\sum_{x} R(x) \log \frac{R(x)}{S(x)} .
\end{aligned}
$$

Here $M=(P+Q) / 2$ denotes the central probability mass function and $\mathrm{KL}$ denotes $\mathrm{KL}$ divergence.
Properly estimating the parameters for the RBF kernel is particularly important in order to ensure proper learning [8]. We used a particle swarm ensemble clustering algorithm called the ERCE algorithm for this purpose [10]. It has been argued that the algorithm can estimate the number of clusters directly from the data using swarm intelligence and ensemble aggregation [10], [16].

Using ERCE, the support vector centroids $\boldsymbol{\mu}_{k} \in$ $\left\{\boldsymbol{\mu}_{1}, \boldsymbol{\mu}_{2}, \ldots\right\}$ as well as the kernel radius $\sigma_{k} \in\left\{\sigma_{1}, \sigma_{2}, \ldots\right\}$ can be inferred from the training data. The steps are as follows:

1) Execute ERCE [10] to cluster the training set $\mathbb{X}_{\text {train }}=$ $\left\{\mathbf{x}_{1}, \mathbf{x}_{2}, \ldots\right\}$ to an arbitrary number of cluster based on JS distance (i.e. the square root of JS divergence).

2) Aggregate the ensemble clustering results using average linkage to get the final clustered sets $\left\{\mathbb{C}_{1}, \mathbb{C}_{2}, \ldots, \mathbb{C}_{K}\right\}$, where $K$ is determined automatically by ERCE at ensemble aggregation. The corresponding centroid vector $\boldsymbol{\mu}_{k} \in\left\{\boldsymbol{\mu}_{1}, \ldots, \boldsymbol{\mu}_{K}\right\}$ is computed as conditional expectation as follows,

$$
\boldsymbol{\mu}_{k}=\mathrm{E}\left[\mathbf{x} \mid \mathbb{C}_{k}\right]=\frac{1}{\left|\mathbb{C}_{k}\right|} \sum_{\mathbf{x} \in \mathbb{C}_{k}} \mathbf{x} .
$$

3) The RBF kernel radius for the $k^{t h}$ support vector is taken as the square root of conditional JS divergence as follows,

$$
\sigma_{\mathrm{k}}^{2}=\mathrm{E}\left[\mathrm{JS}\left(\mathbf{x} \| \boldsymbol{\mu}_{k}\right) \mid \mathbb{C}_{k}\right]=\frac{1}{\left|\mathbb{C}_{k}\right|} \sum_{\mathbf{x} \in \mathbb{C}_{k}} \operatorname{JS}\left(\mathbf{x} \| \boldsymbol{\mu}_{k}\right) .
$$

4) Given the optimized RBF kernel parameters, the SVM is then trained using the SMO algorithm.

\section{RESUlTS AND Discussion}

Table I summarizes the results from from 30 repetitions of SVM training and testing episodes using 5 subbands $\left(\mathrm{SB}_{5}\right)$ and 3 subbands $\left(\mathrm{SB}_{3}\right)$, as well as $32\left(\mathrm{CH}_{32}\right)$ and $18\left(\mathrm{CH}_{18}\right)$ EEG channels.

We used daubechies $5(\mathrm{db} 5)$ as the mother wavelet. The wavelet features are computed from the detail coefficients:

- $5^{\text {th }}$ detail coefficients: $x_{D_{5}} \approx$ Delta $(3-4 \mathrm{~Hz})$;

- $4^{\text {th }}$ detail coefficients: $x_{D_{4}} \approx$ Theta $(5-8 \mathrm{~Hz})$;

- $3^{\text {rd }}$ detail coefficients: $x_{D_{3}} \approx$ Alpha $(9-16 \mathrm{~Hz})$;

- $2^{\text {nd }}$ detail coefficients: $x_{D_{2}} \approx \operatorname{Beta}(17-32 \mathrm{~Hz})$; and

- $1^{\text {st }}$ detail coefficients: $x_{D_{1}} \approx$ Gamma $(33-64 \mathrm{~Hz})$.

Table I shows that the emotion classification using wavelet entropy generally have higher accuracy than relative wavelet energy. Using only the alpha, beta, and gamma subbands $\left(\mathrm{SB}_{3}\right)$ seem to the overall accuracy. Wavelet entropy features up achieved $61.8 \%$ accuracy under the $\mathrm{CH}_{32}, \mathrm{SB}_{3}$ setting.

When the number of channel is reduced to 18 , we observed a slight increase in sensitivity and a substantial drop in specificity. The confusion matrix in Table II may help explain this phenomenon. As observed in Table II, sad emotion was classified up to $88.89 \%$ true positive rate, while the other three emotions achieved lower than $80 \%$ true positive rate. Fig. 4 indicates that these channels are lacking the information to distinguish between angry and happy emotions. 
TABLE I

EXPERIMENTAL RESULT SUMMARY.

\begin{tabular}{lccc}
\hline $\begin{array}{l}\text { Channel, } \\
\text { Subband }\end{array}$ & Sensitivity & Specificity* & Accuracy* \\
\hline & & \\
\hline \multicolumn{4}{l}{ Features: Relative Wavelet Energy } \\
$\mathrm{CH}_{32}, \mathrm{SB}_{5}$ & $72.5 \% \pm 10.6 \%$ & $79.3 \% \pm 7.0 \%$ & $57.3 \% \pm 2.8 \%$ \\
$\mathrm{CH}_{32}, \mathrm{SB}_{3}$ & $75.4 \% \pm 11.1 \%$ & $81.8 \% \pm 5.9 \%$ & $59.6 \% \pm 1.9 \%$ \\
$\mathrm{CH}_{18}, \mathrm{SB}_{3}$ & $73.5 \% \pm 14.8 \%$ & $82.3 \% \pm 6.2 \%$ & $59.6 \% \pm 2.6 \%$ \\
\hline Features: Wavelet Entropy & & \\
$\mathrm{CH}_{32}, \mathrm{SB}_{5}$ & $74.6 \% \pm 10.3 \%$ & $79.8 \% \pm 6.5 \%$ & $59.0 \% \pm 1.7 \%$ \\
$\mathrm{CH}_{32}, \mathrm{SB}_{3}$ & $75.7 \% \pm 12.1 \%$ & $83.7 \% \pm 6.8 \%$ & $61.8 \% \pm 4.4 \%$ \\
$\mathrm{CH}_{18}, \mathrm{SB}_{3}$ & $77.4 \% \pm 14.1 \%$ & $69.1 \% \pm 12.8 \%$ & $60.9 \% \pm 3.2 \%$ \\
\hline
\end{tabular}

* Sensitivity, specificity and accuracy are averaged over the four emotions over 30 experiments.

TABLE II

CONFUSION MATRIX OF THE BEST TRAINED CLASSIFIER USING WAVELET ENTROPY UNDER $\mathrm{CH}_{18}, \mathrm{SB}_{3}$ SETTING.

\begin{tabular}{lccccc}
\hline & & \multicolumn{4}{c}{ Output class } \\
\cline { 3 - 6 } & & Sad & Relaxed & Angry & Happy \\
\hline \multirow{4}{*}{ Target Class } & Sad & $\mathbf{8 8 . 8 9 \%}$ & $00.00 \%$ & $00.00 \%$ & $11.11 \%$ \\
& Relaxed & $28.57 \%$ & $\mathbf{6 4 . 2 9 \%}$ & $00.00 \%$ & $07.14 \%$ \\
& Angry & $09.09 \%$ & $00.00 \%$ & $\mathbf{7 2 . 7 3 \%}$ & $18.18 \%$ \\
& Happy & $09.09 \%$ & $20.00 \%$ & $18.18 \%$ & $\mathbf{7 2 . 7 3 \%}$ \\
\hline
\end{tabular}

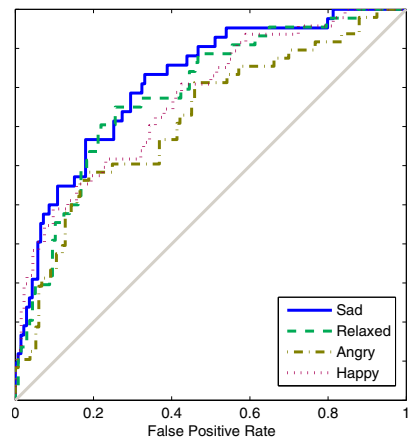

Fig. 3. Receiver Operating Characteristic (ROC) of an average case scenario.

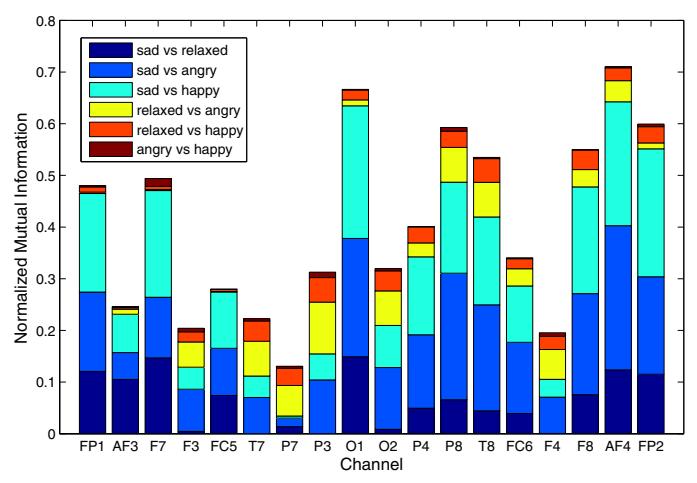

Fig. 4. The Normalized Mutual Information between each channel and each emotions. It can be seen that the wavelet entropy features were predominantly biased towards characterizing sad emotion, whereas relaxed, happy, and angry emotions were seemingly underrepresented.

\section{CONCLUSION}

The proposed EEG emotion recognition system has successfully identified emotional states including happy, sad, angry, and relaxed using wavelet analysis. Using the DEAP [9] we discovered that the alpha, beta, and gamma EEG subbands are particularly important for emotion recognition. Our proposed approach achieved an average sensitivity and specificity of $77.4 \%$ and $69.1 \%$, respectively. The limitation of our method is that transient patterns and quasi-stable states that may exist in the EEG are overlooked in both energy and entropy formulations. Having this in mind, potential research directions that worth considering include incorporating EEG microstates and/or brain connectivity features.

\section{REFERENCES}

[1] J. A. Russell, "A circumplex model of affect." Journal of Personality and Social Psychology, vol. 39, no. 6, pp. 1161-1178, 1980.

[2] C. Torres, A. Orozco, and M. Alvarez, "Feature selection for multimodal emotion recognition in the arousal-valence space," in Engineering in Medicine and Biology Society (EMBC), 2013 35th Annual International Conference of the IEEE, July 2013, pp. 4330-4333.

[3] G. Valenza, L. Citi, A. Lanatá, E. P. Scilingo, and R. Barbieri, "Revealing real-time emotional responses: a personalized assessment based on heartbeat dynamics," Scientific Reports, vol. 4, 2014.

[4] R. Jenke, A. Peer, and M. Buss, "Feature extraction and selection for emotion recognition from eeg," IEEE Trans. Affective Comput., vol. 5, no. 3, pp. 327-339, 2014.

[5] D. Nie, X.-W. Wang, L.-C. Shi, and B.-L. Lu, "Eeg-based emotion recognition during watching movies," in Neural Engineering (NER), 2011 5th International IEEE/EMBS Conference on, April 2011, pp $667-670$.

[6] Rizon, "Discrete wavelet transform based classification of human emotions using electroencephalogram signals," American Journal of Applied Sciences, vol. 7, no. 7, pp. 878-885, 2010.

[7] J.-Y. Zhu, W.-L. Zheng, Y. Peng, R.-N. Duan, and B.-L. Lu, "Eegbased emotion recognition using discriminative graph regularized extreme learning machine," in Neural Networks (IJCNN), 2014 International Joint Conference on, July 2014, pp. 525-532.

[8] M. I. Schlesinger and V. Hlavac, Ten lectures on statistical and structural pattern recognition. Kluwer Academic, 2002.

[9] S. Koelstra, C. Muhl, M. Soleymani, J.-S. Lee, A. Yazdani, T. Ebrahimi, T. Pun, A. Nijholt, and I. Patras, "Deap: A database for emotion analysis ; using physiological signals," IEEE Trans. Affective Comput., vol. 3, no. 1, pp. 18-31, 2012.

[10] M. Yuwono, S. Su, B. Moulton, Y. Guo, and H. Nguyen, "An algorithm for scalable clustering: Ensemble rapid centroid estimation," in Evolutionary Computation (CEC), 2014 IEEE Congress on, July 2014, pp. 1250-1257.

[11] G. Tinguely, L. A. Finelli, H.-P. Landolt, A. A. Borbély, and P. Achermann, "Functional eeg topography in sleep and waking: Statedependent and state-independent features," NeuroImage, vol. 32, no. 1, pp. 283-292, 2006.

[12] A. Handojoseno, J. Shine, T. Nguyen, Y. Tran, S. Lewis, and $\mathrm{H}$. Nguyen, "The detection of freezing of gait in parkinson's disease patients using eeg signals based on wavelet decomposition," in Engineering in Medicine and Biology Society (EMBC), 2012 Annual International Conference of the IEEE, Aug 2012, pp. 69-72.

[13] O. A. Rosso, S. Blanco, J. Yordanova, V. Kolev, A. Figliola, M. Schürmann, and E. Başar, "Wavelet entropy: a new tool for analysis of short duration brain electrical signals," Journal of Neuroscience Methods, vol. 105, no. 1, pp. 65-75, 2001.

[14] C. E. Shannon, "A mathematical theory of communication," Bell system technical journal, vol. 27, 1948.

[15] B. Fuglede and F. Topsoe, "Jensen-shannon divergence and hilbert space embedding," in Proceedings. International Symposium on Information Theory (ISIT), 2004., June 2004, pp. 31-

[16] M. Yuwono, "Ensemble rapid centroid estimation: A semi-stochastic consensus particle swarm approach for large scale cluster optimization," Ph.D. dissertation, University of Technology, Sydney (UTS), Australia, March 2015. 